\title{
STRESS FACTOR IN THE DISG SYNDROME
}

\author{
J. C. Scott, Oxford, England \\ From the Nuffield Orthopaedic Centre, Oxford
}

The complexity of the disc syndrome, and the frequency with which we meet it, is perhaps sufficient to justify a slightly unusual approach, and the presentation of a sequence of ideas supported by experimental evidence.

Consider first the sequence of events on the clinical side, which has been-in part at least-responsible for the development of the idea. 1) There is an impression among many of those conducting large clinics that an increased number of people have suffered from the disc syndrome during and since the recent war, with all the symptoms of acute and chronic back pain and root irritation. Statistics relating to the frequency of the conditions are unreliable, because of the popularity of the diagnosis, but any figures produced would strongly support this contention. 2) In general, conditions that have shown a marked increase in the years during and since the war are the virus diseases and the psychosomatic disorders. Perhaps because of this, and perhaps as the last line of defence, there is an increase in the emphasis on the psychological aspect of the problem in the disc syndrome. For example, Barr (1951) has stated: "Low back and sciatic pain signify a state of stimulation of the cerebral cortex, and are not an affection of the back: it is important to study and treat the patient and not just his back." 3) Injury to the back was at first thought to be a direct and frequent causative factor. More recently less importance has been attached to it. Attacks of acute back pain have been known to come on after exposure, strenuous exercise without back injury, and prolonged emotional strain. It seems likely that these influences would be more likely to produce their effect through some general physiological reaction, rather than as a result of any direct effect on the back. 4) There is little doubt that the understanding of the syndrome is inadequate, and the treatment therefore often haphazard and empirical. None of the accepted forms of conservative treatment is universally successful in dealing even with the acute attack expeditiously, let alone having any influence in preventing subsequent attacks. Most of the ardent advocates of surgical treatment are now prepared to admit that it has a limited place in the treatment of the syndrome.

To summarise the points in the clinical hypothesis: The incidence of the condition has greatly increased, as has the emphasis on the psychological or functional aspect of the problem. Attacks have been reported after incidents in which the back was not directly involved, but in which a general psychological reaction had certainly occurred. Accepted methods of treatment leave much to be desired.

It is suggested that a sequence of events occurs in which the pressure within the annulus increases beyond the normal level (in some instances leading to acute back pain) and that this may be repeated until rupture of the annulus occurs in those discs naturally subjected to the greatest pressure. It is further suggested that emotional strain may be a factor in producing the periodic increases in pressure.

There is one significant type of radiographic appearance which may accompany the early attacks of acute back pain. The disc, instead of being its normal shape or narrowed, is expanded-particularly at the centre-and the opposing surfaces of vertebral bodies above and below are rounded to accommodate it (Fig. 1). This change is certainly not a constant finding, but it is one that merits more attention than it has received. The widened disc is associated with the acute attack of back pain, but when root signs have been present for any length of time it is no longer present-though even when the disc is narrowed the

VoL. 37 B, No. 1, FEBRUARY 1955 
"scalloped" vertebral bodies may give evidence of the previous existence of the state of affairs described above.

There is nothing new about the suggestion of altering tension within the disc. Hirsch (1951) made an exhaustive study of the subject. He pointed out that increasing the pressure by injection into the disc reproduces the typical pain; and he found that the changes in the nucleus precede those in the annulus, which habitually ruptures from within outwards.

It has been shown that the disc can take up large quantities of fluid when placed in solutions of low osmotic pressure (Charnley 1951); and that by placing a preparation of fresh post-mortem material, consisting of an intact disc and two vertebral plates, in water or normal saline a very high pressure can be produced within the disc (Naylor and Smare 1953).

It may seem that the above observations bear little relationship to the conditions existing in life, but reference to the work of Opie and Rothbard (1953) suggests that this is not so.

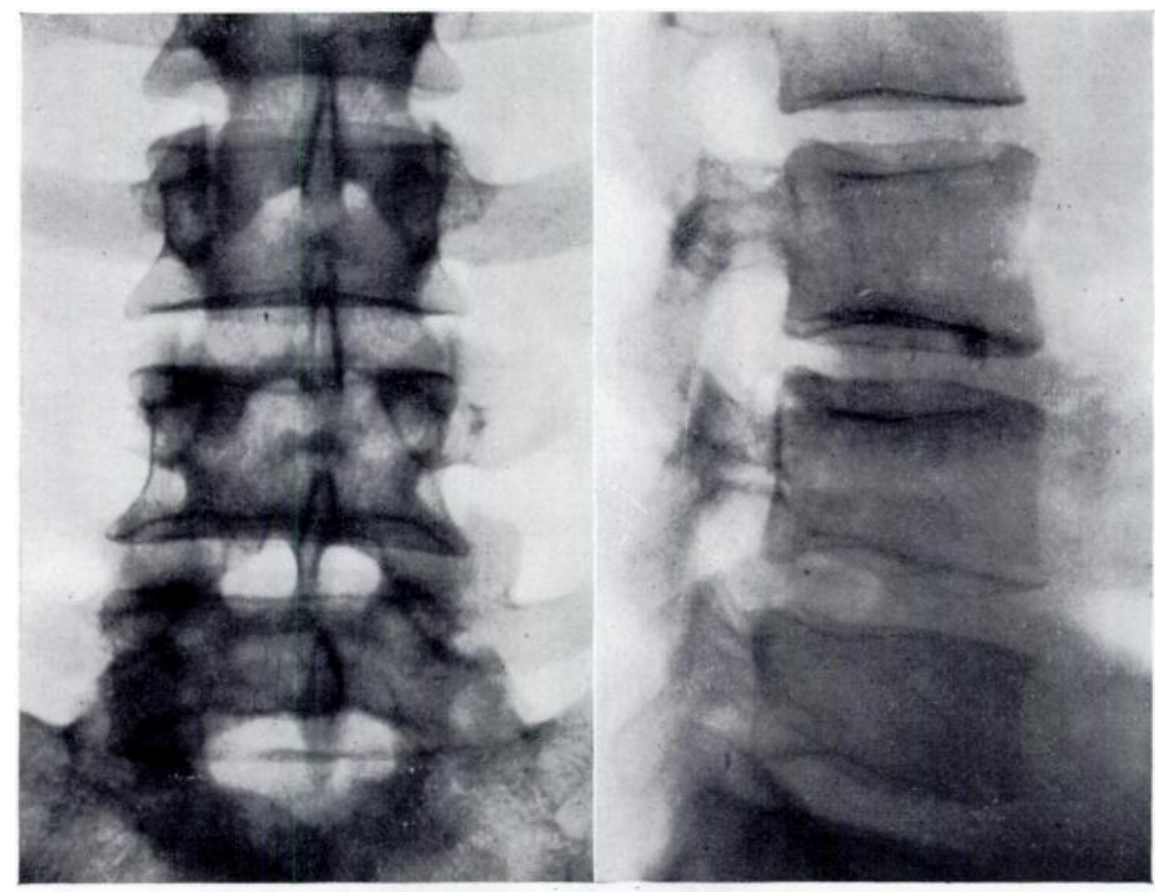

FIG. 1

Central expansion of disc suggested by hollowing of opposing surfaces of vertebral bodies.

The reaction of collagenous tissue exposed to solutions of varying osmotic pressure has been extensively studied, and Opie and Rothbard wrote: "It is noteworthy that dense fibrous tissue, which in view of its structure seems little pervious to the movement of water, is peculiarly adapted to absorb and hold it." It also seems that collagenous tissue, which contains a considerable quantity of mucopolysaccharide, is capable of taking up even larger quantities of fluid. It is somewhat uncertain whether this process is one of osmosis or hydration. There is therefore some evidence that the disc is capable of taking up fluid, that this leads to an increased pressure within the annulus, and that increased pressure leads to pain.

Disturbance of the water balance leading to retention may come about in various ways. Sudden and prolonged emotional strain leads to an increased secretion of vasopressin, the antidiuretic hormone of the pituitary. Adrenalectomy, and the interference with secretion of the adrenal, increases the antidiuretic effect of various systemic pressor substances. In physical trauma (including surgery), sometimes of even a minor degree, there is an increase 
in adrenocortical activity-also an associated alteration in the water balance and retention of electrolytes, particularly sodium and chloride (Le Quesne 1953). It is known also that the administration of A.C.T.H. or cortisone causes a retention of salt and water. There is no reliable evidence as to how this retained fluid is distributed.

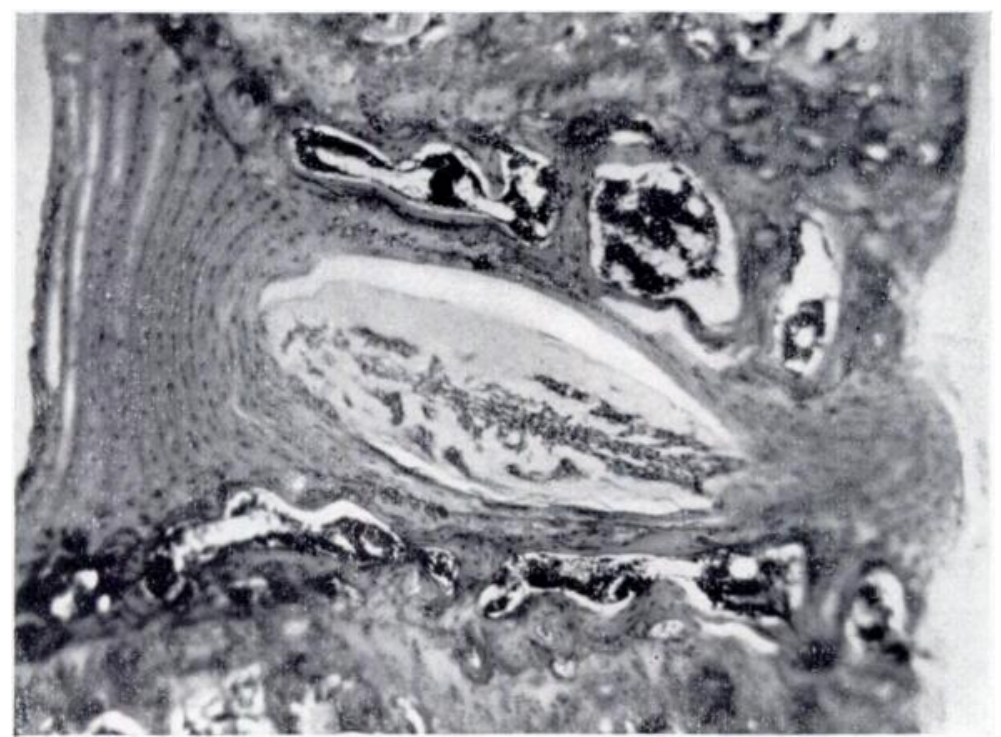

FIG. 2

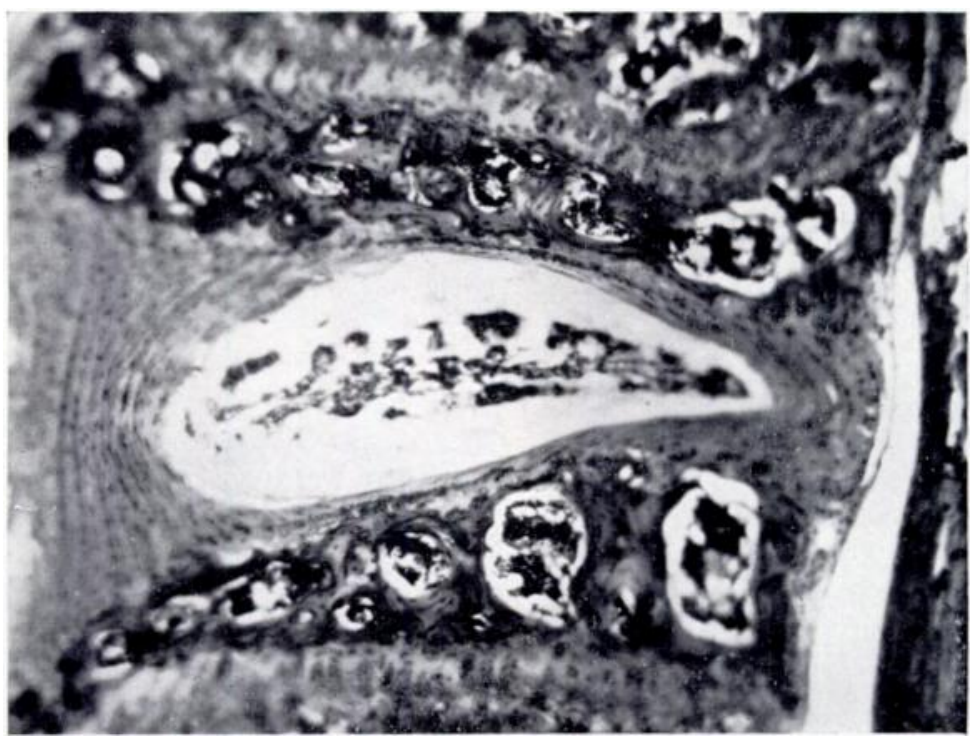

FIG. 3

Typical appearance of disc from control animal (Fig. 2) and experimental animal (Fig. 3).

\section{EXPERIMENTAL METHOD AND RESULTS}

With these things in mind it was decided to investigate, in animals, the effect of stress on the intervertebral disc and the size of the nucleus.

The response of the body to non-specific stress has been studied by many people. Certain changes found in animals in response to stress, and considered to be characteristic of the 
"general adaptation syndrome" described by Selye (1950), include involution of the thymus and enlargement of the adrenals. There seems to be a fairly wide measure of agreement that these changes are evidence of the reaction of the body to stress which may be applied in different ways.

It has been shown (Clarke 1953) that when voles (Microtus agrestis) have been subjected to daily periods of fighting their adrenals increase in weight, the thymus decreases, and the spleen shows a pathological change. In Clarke's experiments the stressor agent was a pair of old voles into whose cage a young animal had been introduced. Organ weights of these young animals were compared with those of littermate controls.

Preliminary tests were carried out at the Bureau of Animal Population, Oxford, by Dr D. H. Chitty, using methods similar to Clarke's, but under less rigidly controlled conditions; for example, the control and experimental animals were not all littermates, but had been selected at random from the males available at the time. These tests will not be described in detail; it need only be said that the experimental voles were in contact with strangers for about two hours on each of three or four days, after which they and their controls were killed. Dr A. H. T. Robb-Smith was kind enough to examine the spines, and a summary of his report is as follows. The specimens consisted of vertical sections of the vertebral columns of eighteen voles - eight control and ten experimental animals. There were no gross histological differences between the two series, although one gained the impression that in the experimental animals the nucleus pulposus showed a greater basophilia. Measurements were made to find out if there was any alteration in the volume of the nucleus pulposus, by determining the ratio between the total disc area and the area of the nucleus pulposus. Seventy-nine discs were measured, and it was found that in the control series the mean of the ratios of the disc area to nucleus pulposus area was 2.2304 , with a range from 1.62 to 3.50 ; in the experimental series the mean was 1.9041, with a range from 1.35 to 3.35. Dr Rosemary Biggs examined these figures statistically and found that the difference of the means of the two series was $0 \cdot 3263$. By the " $t$ " test, " $t$ " $=\mathbf{3 . 0 2 5 6}$, which is highly significant, the probability of obtaining this value by chance being between $1: 100$ and $1: 1,000$. Thus it appeared that in the experimental animals the area of the nucleus pulposus in relation to the total disc area was larger than in the control animals, the difference being statistically significant.

Because of the encouraging results of these preliminary experiments it was decided to repeat the experiment using only littermates, and excluding all possible sources of error. The size of nucleus relative to the total area of the intervertebral disc was estimated by the same method as that used in the previous studies. One hundred and thirty-one discs in twenty-four spines were measured, the measurer having no knowledge as to which were controls and which experimental animals (Figs. 2 and 3). Statistical analysis of these measurements showed a highly significant increase in the relative size of the nucleus proportional to the changes in adrenals, thymus and spleen. The details of these experiments and their results are being prepared for publication. They provide evidence that the reaction to "stress" which leads to certain changes in adrenals, thymus and spleen-thought to be due to increased adrenocortical activity-also leads to an increase in the size of the nucleus pulposus (Chitty et al. (MS.)).

\section{COMMENT}

It is obvious that these results do no more than add a little corroboration to an idea which has developed as a result of clinical observation. They do not of themselves make any direct contribution, but it is just possible that they point in a direction which may be of some value in relation to etiology and treatment, and which has not so far been adequately investigated.

I should like to acknowledge the valuable assistance of Dr A. H. T. Robb-Smith in the preparation of this paper, and to thank Dr Rosemary Biggs for the statistical analysis of the first series. 


\section{REFERENCES}

BARR, J. S. (1951): Low Back Pain and Sciatica. Journal of Bone and Joint Surgery, 33-B, 469.

Charnley, J. (1951): Fluid Imbibition as a Cause of Herniation of the Nucleus Pulposus. Journal of Bone and Joint Surgery, 33-B, 472.

Chitty, D., Chitty, H., Leslie, P. H., and Scott, J. C. (MS.): Changes in the Relative Size of the Nucleus in the Intervertebral Disc of Stressed Orkney Voles.

Clarke, J. R. (1953): The Effect of Fighting on the Adrenals, Thymus and Spleen of the Vole (Microtus Agrestis). Journal of Endocrinology, 9, 114.

Hirsch, C. (1951): Morbid Anatomy of Lumbar Discs. Journal of Bone and Joint Surgery, 33-B, 472.

Naylor, A., and Smare, D. L. (1953): Fluid Content of the Nucleus Pulposus as a Factor in the Disk Syndrome: Preliminary Report. British Medical Journal, ii, 975.

OpIE, E. L., and Rothbard, M. B. (1953): Water Exchange of Collagenous Tissues and of Gelatin. Journal of Experimental Medicine, 97, 499.

Quesne, L. P. Le (1953): The Administration of Water and Electrolytes During the Post-Operative Period. Annals of the Royal College of Surgeons of England, 13, 224.

SELye, H. (1950): The Physiology and Pathology of Exposure to Stress. Montreal: Acta, Inc., Medical Publishers. 